\title{
Freeze-Thaw and Cold-Shock Resistance of Saccharomyces cerevisiae as Affected by Plasma Membrane Lipid Composition
}

\author{
By PETER H. CALCOTT† AND ANTHONY H. ROSE* \\ Zymology Laboratory, School of Biological Sciences, University of Bath, Bath, Avon, U.K.
}

(Received 15 July 1981)

\begin{abstract}
Populations of Saccharomyces cerevisiae NCYC 366, grown anaerobically under conditions that lead to enrichment of membranes with specific sterols and fatty-acyl residues, were subjected to freeze-thaw cycles and cold-shock stress. One freeze-thaw cycle caused considerable loss in viability, the loss being greater with organisms enriched in campesterol or cholesterol rather than ergosterol or stigmasterol. Retention of viability was greater in populations enriched in linoleyl rather than oleyl residues. Organisms enriched in ergosterol and either oleyl or cetoleyl residues were equally susceptible to death following freeze-thaw cycles, but less so than organisms enriched in palmitoleyl residues. Speed of freezing had little effect on retention of viability in organisms enriched in ergosterol and linoleyl residues, but an effect was observed in populations enriched in cholesterol and linoleyl residues. In populations enriched in ergosterol and linoleyl residues, sodium chloride protected against loss of viability, whereas in organisms enriched in cholesterol and linoleyl residues the salt rendered populations more susceptible to death following freezing and thawing. Resistance to cold-shock stress was also greater in populations enriched in ergosterol or stigmasterol rather than campesterol or cholesterol, and with organisms enriched in linoleyl rather than oleyl residues.
\end{abstract}

\section{INTRODUCTION}

Microbial plasma membranes play an important role in determining resistance to freeze-thaw and cold-shock stresses (Mazur, 1970; Ray \& Speck, 1973; MacLeod \& Calcott, 1976; Calcott, 1978). However, few reports have implicated specific components of the membrane. Smittle et al. (1974), in an examination of the survival of strains of Lactobacillus bulgaricus following exposure to freeze-thaw cycles, concluded that a high proportion of $\mathrm{C}_{19}$ cyclopropane fatty-acyl residues in cellular lipids favoured survival. However, this study was based on a comparison of genetically different strains. Using unsaturated fatty acid-requiring auxotrophs, Haest et al. (1972) assessed the effect of membrane fatty-acyl composition in Escherichia coli on release of potassium ions following imposition of a cold shock. They concluded that cation release started at the temperature where phase transitions occur in the paraffin core of the membrane. Finally, Lee et al. (1977) have shown that the cytochrome content of bacterial plasma membranes is related to sensitivity to sodium chloride on freezing and thawing.

The present paper extends these studies to the eukaryote Saccharomyces cerevisiae, and reports that both the sterol and fatty-acyl composition of the plasma membrane are important for this organism to remain viable after being subjected to freeze-thaw cycles or cold shock. The study exploits the anaerobically-induced requirements for a sterol and an unsaturated fatty acid (Andreasen \& Stier, 1953, 1954). Both of these nutritional requirements are fairly

\footnotetext{
$\dagger$ Permanent address: CR-Bioproducts Laboratory, The Dow Chemical Company, Midland, Michigan 48640, U.S.A.
} 
broad (Light et al., 1962; Proudlock et al., 1968), and this permits specific enrichments to be made in the sterol (Hossack \& Rose, 1976) and fatty-acyl (Thomas et al., 1978) composition of the yeast plasma membrane, without changing the content or composition of other types of lipid in the organism (Rose, 1977).

\section{METHODS}

Experimental cultures. Saccharomyces cerevisiae NCYC 366 was maintained on slopes of malt extract/yeast extract/glucose/mycological peptone/agar (Wickerham, 1951). Cultures were grown aerobically as described by Patching \& Rose (1969) in one-litre batches of medium (pH 4.5) containing (per litre) $100 \mathrm{~g}$ glucose, $3 \mathrm{~g}$ $\left(\mathrm{NH}_{4}\right)_{2} \mathrm{SO}_{4}, 3 \mathrm{~g} \mathrm{KH}_{2} \mathrm{PO}_{4}, 1 \mathrm{~g}$ yeast extract (Oxoid), $25 \mathrm{mg} \mathrm{MgCl}$ and $25 \mathrm{mg} \mathrm{CaCl}$. The yeast was grown anaerobically in the same medium supplemented with a sterol $\left(5 \mathrm{mg} \mathrm{l}^{-1}\right)$ and an unsaturated fatty acid $\left(30 \mathrm{mg} \mathrm{l}^{-1}\right)$ as described by Alterthum \& Rose (1973). Growth was followed by measuring turbidity at $600 \mathrm{~nm}$. Turbidity values were related to dry wt equiv. by a standard curve. Organisms were harvested from late-exponential phase cultures (240-260 mg dry wt equiv. $\left.1^{-1}\right)$ by centrifugation at $4{ }^{\circ} \mathrm{C}$, and were washed with $67 \mathrm{mM}-\mathrm{KH}_{2} \mathrm{PO}_{4}(\mathrm{pH}$ 4.5).

Stressing of organisms. Populations $\left(10^{7}\right.$ colony-forming units $\mathrm{ml}^{-1}$ ) in $10 \mathrm{ml}$ water or $0.1 \mathrm{M}-\mathrm{NaCl}$ were subjected to freeze-thaw cycles with rapid freezing $\left(100^{\circ} \mathrm{C} \mathrm{min}^{-1}\right)$ by dipping into liquid nitrogen in a $50 \mathrm{ml}$ conical flask, and then thawing. After $5 \mathrm{~min}$ at $-196^{\circ} \mathrm{C}$, samples were thawed slowly $\left(10-20^{\circ} \mathrm{C} \mathrm{min}^{-1}\right)$ by immersion in a waterbath $\left(30^{\circ} \mathrm{C}\right)$ as described by Calcott \& MacLeod $(1974 a)$. Portions were removed for viability determinations after one, two and three freeze-thaw cycles. Populations were frozen slowly by placing a tube containing $1 \mathrm{ml}$ suspension in a deep freeze at $-20^{\circ} \mathrm{C}$ for $1 \mathrm{~h}$. The suspension was then placed in liquid nitrogen for $10 \mathrm{~min}$ before being thawed (Calcott \& MacLeod, 1974a). Populations were frozen extremely rapidly $\left(6000^{\circ} \mathrm{C} \mathrm{min}^{-1}\right)$ by dripping the suspension into liquid nitrogen, before thawing (Calcott \& MacLeod, 1974a). Populations were subjected to cold shock by suspending organisms at $0.25 \mathrm{mg}$ dry wt equiv. $\mathrm{ml}^{-1}$ in 67 mM- $\mathrm{KH}_{2} \mathrm{PO}_{4}$ at room temperature, and diluting the suspension into $67 \mathrm{mM}-\mathrm{KH}_{2} \mathrm{PO}_{4}$ at $4{ }^{\circ} \mathrm{C}$ while the suspension was continuously stirred. Control suspensions were diluted into $67 \mathrm{mM}-\mathrm{KH}_{2} \mathrm{PO}_{4}$ maintained at room temperature. Portions were removed from the diluted suspension for viability measurements at the times indicated.

Viability measurements. The viability of stressed and unstressed populations was measured by diluting in 67 mM- $\mathrm{KH}_{2} \mathrm{PO}_{4}$ at room temperature to give approximately $100-300$ colony-forming units $\mathrm{ml}^{-1}$, and surface plating portions on to Wickerham's (1951) medium solidified with $20 \mathrm{~g}_{\text {agar }} 1^{-1}$. Plates were incubated to constant counts at $30^{\circ} \mathrm{C}$. The percentage viability of stressed populations is indicated with reference to unstressed control populations.

Chemicals. All chemicals used were Analar or of the highest grade available commercially. Cholesterol, ergosterol, stigmasterol, palmitoleic acid ( $\Delta^{9}$ cis-hexadecenoic acid), oleic acid ( $\Delta^{9}$ cis-octadecenoic acid), linoleic acid $\left(\Delta^{9,12}\right.$ cis-octadecadienoic acid) and cetoleic acid ( $\Delta^{11}$ cis-eicosaenoic acid) were purchased from Sigma. Campesterol was a generous gift from $\mathrm{H}$. W. Kircher.

\section{RESULTS}

\section{Freeze-thaw stress}

After being subjected to the first of three freeze-thaw stresses, with rapid freezing, populations of organisms enriched by anaerobic growth with any of the combinations of sterol and unsaturated fatty-acyl residue lost a high proportion of viability (Fig. 1). The loss was, however, considerably greater with organisms containing membranes enriched in campesterol or cholesterol rather than ergosterol or stigmasterol. Moreover, with the exception of organisms enriched in cholesterol, loss of viability was somewhat less in populations suspended in sodium chloride solution than in water. Retention of viability was, furthermore, slightly greater with organisms enriched in linoleyl rather than oleyl residues, again with the exception of organisms enriched in cholesterol. There was no loss of viability in suspensions of organisms in water or sodium chloride solution not subjected to freeze-thaw cycles. The protective effect of sodium chloride was also observed when organisms enriched by anaerobic growth in ergosterol, and in either palmitoleyl or cetoleyl residues, were submitted to the same regime of freeze-thaw cycles (Fig. 2). However, organisms enriched in palmitoleyl residues were more susceptible to the stress than those enriched in either oleyl 

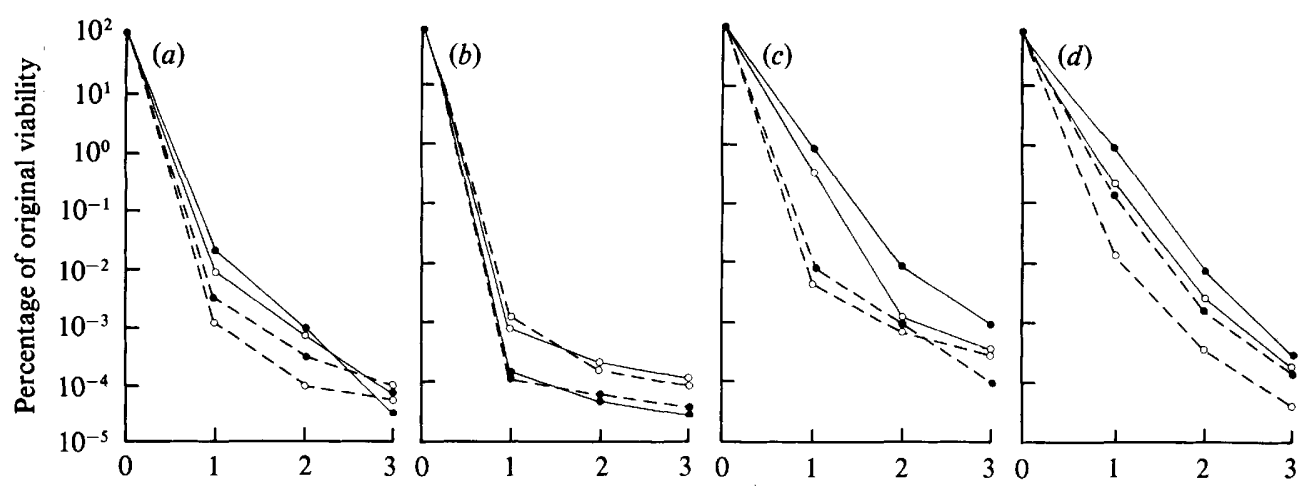

Number of freeze-thaw cycles

Fig. 1. Effect of freeze-thaw cycles with rapid freezing on the percentage viability of Saccharomyces cerevisiae NCYC 366 grown anaerobically with plasma membranes enriched in campesterol $(a)$, cholesterol $(b)$, ergosterol $(c)$ or stigmasterol $(d)$ and either oleyl $(O)$ or linoleyl $(O)$ residues, and suspended in $0.1 \mathrm{M}-\mathrm{NaCl}$ (continuous lines) or water (dashed lines). The values plotted are the average of at least three independent determinations. The maximum variation on any value was $\pm 0.4 \log$ unit of viability. Freeze-thaw stress was applied to populations as described in Methods.

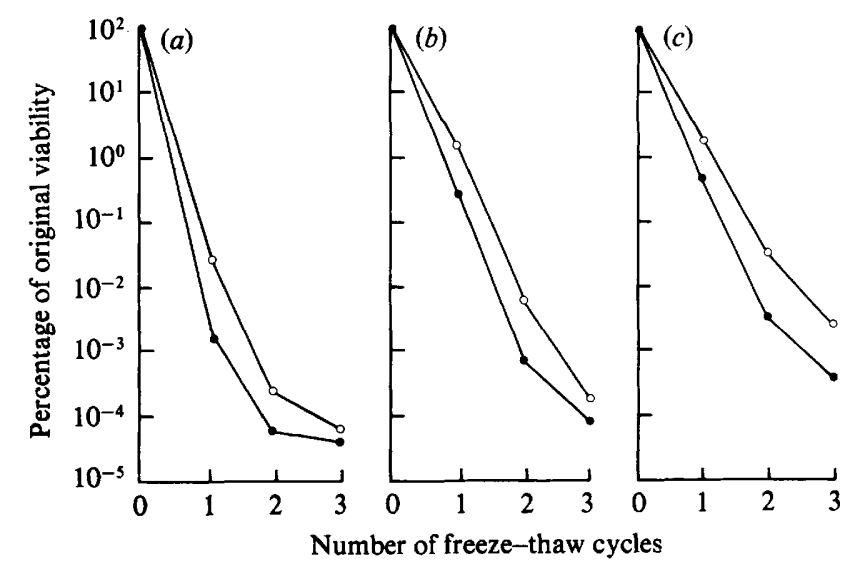

Fig. 2. Effect of freeze-thaw cycles with rapid freezing on the percentage viability of Saccharomyces cerevisiae NCYC 366 grown anaerobically with plasma membranes enriched in ergosterol and palmitoleyl $(a)$ or cetoleyl $(b)$ residues, or grown aerobically $(c)$. Organisms were suspended in $0.1 \mathrm{M} \mathrm{NaCl}(\mathrm{O})$ or water (O). For other details see legend to Fig. 1 .

(Fig. 1) or cetoleyl (Fig. 2) residues. Aerobically grown organisms responded similarly to those enriched in ergosterol and either oleyl or cetoleyl residues (Fig. 2).

Further evidence for the protective effect of sodium chloride was obtained when organisms, enriched in cholesterol or ergosterol and in linoleyl residues, were subjected to one freeze-thaw cycle by the slow, rapid or extremely rapid regimes (Fig. 3). With organisms enriched in ergosterol, the speed at which populations were frozen, in either water or sodium chloride solution, had little effect on the percentage of organisms that remained viable. However, for organisms enriched in cholesterol, slow freezing was more injurious than either of the two more rapid regimes, irrespective of the nature of the suspending liquid. 


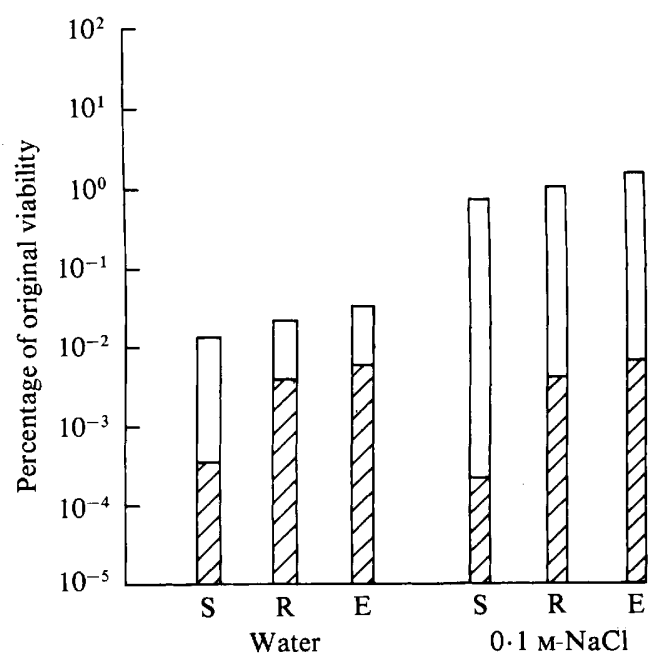

Fig. 3. Histograms showing the decline in percentage viability of Saccharomyces cerevisiae NCYC 366 grown anaerobically with plasma membranes enriched in linoleyl residues and ergosterol (total histogram) or cholesterol (hatched part of histogram) after being subjected to one freeze-thaw cycle using the slow (S), rapid (R) or extrenely rapid (E) regime. Techniques used for applying the stresses are described in Methods. Organisms were suspended in water or $0.1 \mathrm{M}-\mathrm{NaCl}$ as indicated.
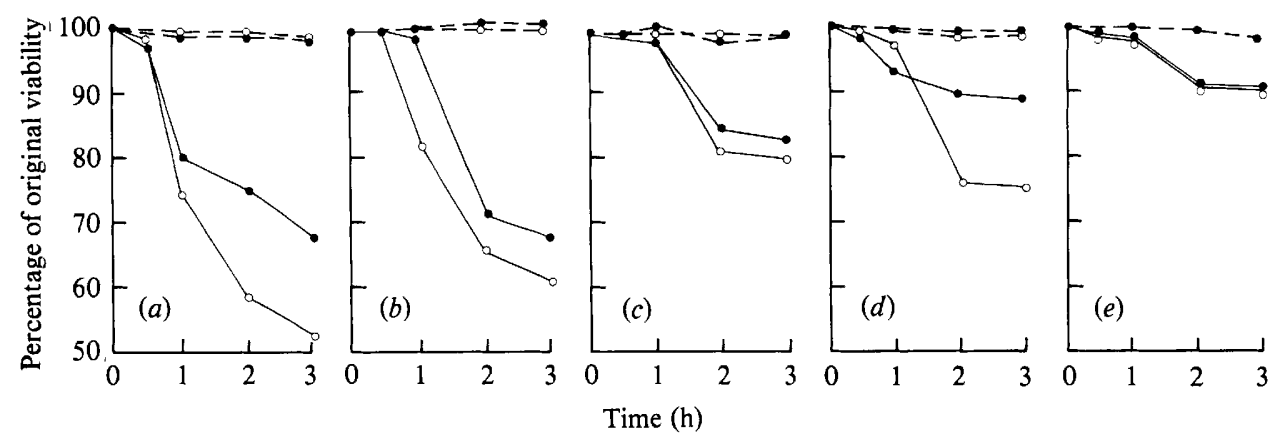

Fig. 4. Time course of loss in percentage viability after submitting Saccharomyces cerevisiae NCYC 366 to cold-shock stress. Organisms were grown anaerobically in media supplemented with oleic $(O)$ or linoleic $(O)$ acid and with campesterol $(a)$, cholesterol $(b)$, ergosterol $(c)$ or stigmasterol $(d)$, or they were grown aerobically $(e)$. The dashed lines indicate the response of unstressed populations. The values plotted are the average of at least three independent determinations. The maximum variation on any value was $\pm 0.4 \mathrm{log}$ unit of viability. The cold shock stress was applied to populations as described in Methods.

\section{Cold-shock stress}

Resistance to a cold-shock stress was also greater in organisms enriched in ergosterol or stigmasterol rather than campesterol or cholesterol (Fig. 4). Enrichment with linoleyl rather than oleyl residues also gave a measure of protection against loss of viability in populations subjected to cold shock, the effect being particularly noticeable in populations of organisms enriched in campesterol or stigmasterol. Aerobically grown organisms were more resistant to cold shock than any of the populations of organisms enriched by anaerobic growth (Fig. 4).

\section{DISCUSSION}

Considerable evidence is available to show that damage occurs to both the plasma membrane and the wall when organisms are subjected to freezing and thawing (MacLeod \& 
Calcott, 1976; Calcott, 1978). The differences observed in the responses of $S$. cerevisiae NCYC 366 with different plasma membrane lipid compositions are probably attributable solely to variations in the lipid components of the membrane, since analyses of walls of organisms grown anaerobically under conditions that enrich the plasma membrane with different sterols and fatty-acyl residues revealed that contents of the major components (glucans, mannan, chitin and protein) do not differ (J. A. Hossack \& A. H. Rose, unpublished observations). Sterols have for a long time been known to condense phospholipid monolayers (Harrison \& Lunt, 1980), and more recent evidence indicates that the degree of interaction between sterol and phospholipid molecules varies with the structure of the sterol side chain. In a study of the capacity to resist stretching of sphaeroplasts of $S$. cerevisiae NCYC 366 having plasma membranes enriched in different sterols and with oleyl residues, Hossack \& Rose (1976) found that resistance was greater when the membranes contained a sterol with an unsaturated side chain (ergosterol or stigmasterol) rather than a saturated side chain (campesterol or cholesterol). They attributed this to a stronger interaction between the unsaturated sterol side chains and methylene groups in fatty-acyl residues of phospholipids, leading to formation of a 'tighter' membrane. The responses of S. cerevisiae NCYC 366 to freeze-thaw stress suggest that a strong interaction between sterol side chains and phospholipids in the plasma membranes confers a measure of protection against loss of viability. The protection offered by stigmasterol was somewhat greater than that of ergosterol, and campesterol more than that of cholesterol, which could indicate that bulkiness of the sterol side chain (stigmasterol has an ethyl, and ergosterol and campesterol a methyl group, while cholesterol has no substituent in the side chain) also offers a degree of protection. This might also explain the diminished protection offered by palmitoleyl as compared with oleyl or cetoleyl residues, for the presence of $\mathrm{C}_{16}$ residues would decrease bulkiness within the membrane. The additional protective effect of linoleyl compared with oleyl residues is probably attributable to the lower temperature at which linoleyl residues undergo transition from the liquid to the gel phase.

There are two types of mechanism to account for protection against loss of viability following freezing and thawing. The protective effect may be observed at all freezing rates, as with the protective effect of glycerol on $E$. coli (Calcott \& MacLeod, 1974a). Alternatively, it may operate only at specific freezing rates, possibly by altering the optimum cooling rate, as with the nutritionally induced protective effect on $E$. coli (Calcott \& MacLeod, 1974b). The protective effect of ergosterol, as compared with cholesterol, in S. cerevisiae was observed at three very different freezing rates, which suggest that an intrinsic property of the plasma membrane is responsible. However, since the effect was more pronounced at slow freezing rates, it is possible that an alteration in the optimum freezing rate could be partly responsible. Mazur $(1963,1970)$ envisaged the optimum freezing rate to be that which allows release of cell water without internal ice formation, but which does not expose the dehydrated cell interior to high concentrations of solutes for long periods. The value for the optimum rate depends on three properties, namely the ratio of surface area to volume of the organisms, the permeability of the plasma membrane to water, and the temperature coefficient of the permeability factor (Mazur, 1963). The shape and volume of cells of S. cerevisiae NCYC 366 grown anaerobically in the presence of different sterols and unsaturated fatty acids do not differ significantly (J. A. Hossack \& A. H. Rose, unpublished observations; Hossack \& Rose, 1976), which suggests that the differences in susceptibility to freezing and thawing of various populations of $S$. cerevisiae are attributable to either or both of the last two factors. It might be expected that 'tightness' in the plasma membrane would decrease the permeability of the membrane to water, which could explain the protective effects of stigmasterol and ergosterol compared with campesterol and cholesterol. Moreover, the presence of linoleyl rather than oleyl residues on membrane phospholipids could influence the temperature coefficient of the permeability factor.

Several workers have demonstrated that inclusion of sodium chloride and other salts in the 
freezing suspension decreases the survival of many micro-organisms after freezing and thawing (MacLeod \& Calcott, 1976; Calcott, 1978). However, Mazur (1966) reported that $0.15 \mathrm{M}$-sodium chloride in the suspending medium protects $S$. cerevisiae from freezing damage to a limited extent over a wide range of freezing rates. This effect was confirmed in the present study, except when organisms had membranes enriched in cholesterol, when susceptibility to freeze-thaw damage was potentiated. The plasma membrane in $S$. cerevisiae is virtually impermeable to the chloride ion (Rothstein, 1972; M. H. J. Keenan \& A. H. Rose, unpublished observation), and the largely unexplained protective effect of sodium chloride could well be caused by some action, ionic or osmotic, on the outside of the plasma membrane. The detrimental effect of sodium chloride with organisms enriched in cholesterol may result from entry of chloride ions into organisms following extensive permeability changes in the plasma membrane.

Loss of viability in yeast following imposition of a cold shock stress has not previously been reported (MacLeod \& Calcott, 1976). The percentage loss of viability when a population of aerobically grown organisms was submitted to cold shock in the present study was small, especially over the first hour. However, anaerobically grown organisms were more susceptible to the stress irrespective of the nature of the lipid enrichment, and most so when the sterol had a saturated side chain. This suggests that a strong interaction between sterol side chains and phospholipid molecules in the plasma membrane may also confer a measure of protection against cold shock. Moreover, the protective effect of linoleyl compared with oleyl residues is in agreement with the findings of Haest et al. (1972) with unsaturated fatty acid-requiring auxotrophs of $E$. coli, and further supports the view that much of the damage caused by cold shock occurs after the occurrence of a phase change in plasma membrane lipids.

This research was supported by a grant to P.H.C. from the U.S. Army Research Office (Grant No. DRXRO-CB-15525-L) and to A.H.R. from the Science Research Council (U.K.) (Grant No. GR/A/41564) for which we express our gratitude.

\section{REFERENCES}

Alterthum, F. \& Rose, A. H. (1973). Osmotic lysis of sphaeroplasts from Saccharomyces cerevisiae grown anaerobically in media containing different unsaturated fatty acids. Journal of General Microbiology 77, 371-382.

Andreasen, A. A. \& Stier, T. H. B. (1953). Anaerobic nutrition of Saccharomyces cerevisiae. I. Ergosterol requirement for growth in a defined medium. Journal of Cellular and Comparative Physiology 41, 23-36.

Andreasen, A. A. \& Stier, T. J. B. (1954). Anaerobic nutrition of Saccharomyces cerevisiae. II. Unsaturated fatty acid requirement for growth in a defined medium. Journal of Cellular and Comparative Physiology 43, 271-281.

CalcotT, P. H. (1978). Freezing and Thawing Microbes. Shildon, Co. Durham: The Meadowfield Press.

Calcott, P. H. \& MacLeod, R. A. (1974a). Survival of Escherichia coli from freeze-thaw damage: a theoretical and practical study. Canadian Journal of Microbiology 20, 671-681.

Calcott, P. H. \& MacLeod, R. A. (1974b). Survival of Escherichia coli from freeze-thaw damage: influence of nutritional status and growth rate. Canadian Journal of Microbiology 20, 683-689.

Haest, C. W. M., De Gier, J., Van Es, G. A., VerkleiJ, A. J. \& VAN DeEnen, L. L. M. (1972).
Fragility of the permeability barrier of Escherichia coli. Biochimica et biophysica acta 288, 43-53.

HARrison, R. \& LUNT, G. G. (1980). Biological Membranes, 2nd edn. Glasgow: Blackie.

Hossack, J. A. \& Rose, A. H. (1976). Fragility of plasma membranes in Saccharomyces cerevisiae enriched with different sterols. Journal of Bacteriology 127, 67-75.

Lee, S. K., CalcotT, P. H. \& Macleod, R. A. (1977). Relationship of cytochrome content to the sensitivity of bacteria to sodium chloride and freezing and thawing. Canadian Journal of Microbiology 23, 413-419.

Light, R. J., LennaRz, W. J. \& Bloch, K. (1962). The metabolism of hydroxystearic acids in yeast. Journal of Biological Chemistry 237, 1793-1800.

Macleod, R. A. \& CalcotT, P. H. (1976). Cold shock and freezing damage to microbes. Symposia of the Society for General Microbiology 26, 81-109.

MAZUR, P. (1963). Kinetics of water loss from cells at sub-zero temperatures and the likelihood of intracellular freezing. Journal of General Physiology 47, 347-369.

Mazur, P. (1966). Physical and chemical basis of injury in single-celled micro-organisms subjected to freezing and thawing. In Cryobiology, pp. 214-315. Edited by H. T. Meryman. New York: Academic Press. 
Mazur, P. (1970). Cryobiology: the freezing of biological systems. Science 168, 939-949.

Patching, J. W. \& Rose, A. H. (1969). The effects and control of temperature. Methods in Microbiology 2, 23-38.

Proudlock, J. W., Wheeldon, L. W., Jollow, D. J. \& Linnane, A. W. (1968). Role of sterols in Saccharomyces cerevisiae. Biochimica et biophysica acta 152, 434-437.

RAY, B. \& SPECK, M. L. (1973). Freeze-injury in bacteria. CRC Critical Reviews in Clinical Laboratory Sciences 4, 161-213.

Rose, A. H. (1977). Dialling the composition of the yeast plasma membrane. In Alcohol, Industry and Research, pp. 179-189. Edited by O. Forsander, K. Eriksson, E. Oura \& P. Jounela-Eriksson. Helsinki: State Alcohol Monopoly.
RothsteIN, A. (1972). Ion trạsport in microorganisms. In Metabolic Pathways, vol. VIA, pp. 17-39. Edited by L. E. Hokin. New York: Academic Press.

Smittle, R. B., Gilliland, S. E., Speck, M. L. \& WALTER, W. M., JR (1974). Relationship of cellular fatty acid composition to survival of Lactobacillus bulgaricus in liquid nitrogen. Applied Microbiology 27, 738-743.

Thomas, D. S., Hossack, J. A. \& Rose, A. H. (1978), Plasma-membrane lipid composition and ethanol tolerance in Saccharomyces cerevisiae. Archives of Microbiology 117, 239-245.

WickerhaM, L. J. (1951). Taxonomy of yeasts. 1. Techniques of classification. United States Department of Agriculture Technical Bulletin No. 1029. 